
\title{
3 Research Suare \\ Exosomal miR-3180-3p Inhibits Proliferation and Metastasis of Non-Small Cell Lung Cancer by Downregulating FOXP4
}

\section{Tengfei Chen}

First Affiliated Hospital of Soochow University

Yali Liu

Soochow University

Chang Li

First Affiliated Hospital of Soochow University

Chun Xu

First Affiliated Hospital of Soochow University

Cheng Ding

First Affiliated Hospital of Soochow University

Jun Chen

First Affiliated Hospital of Soochow University

Huifei Zheng

Auburn University

JunZhao ( $\nabla$ junzhao@suda.edu.cn )

First Affiliated Hospital of Soochow University https://orcid.org/0000-0003-3846-8998

\section{Primary research}

Keywords: non-small cell lung cancer, exosomes, miR-3180-3p, FOXP4

Posted Date: September 8th, 2020

DOI: https://doi.org/10.21203/rs.3.rs-64391/v1

License: (1) (1) This work is licensed under a Creative Commons Attribution 4.0 International License.

Read Full License

Version of Record: A version of this preprint was published at Thoracic Cancer on December 21st, 2020. See the published version at https://doi.org/10.1111/1759-7714.13759. 


\section{Abstract}

Background: Non-small cell lung cancer (NSCLC) is the most malignant cancers worldwide, but the pathogenesis is not completely known. In this study, we explored the function and mechanism of exosomes transferring miR-3180-3p in NSCLC progression.

Method: The expression of miR-3180-3p of NSCLC tissues and para-carcinoma tissues was from the GEO database (GEO: GSE53882). The exosomes derived from A549 cells were identified. The proliferation, migration and invasion were measured after treatment with exosomal miR-3180-3p or transfected by miR-3180-3p mimics. The relationship between miR-3180-3p and forkhead box P4 (FOXP4) was predicted by bioinformatics tool and measured dual-luciferase reporter gene assay and western blotting. At last, mouse xenograft model of NSCLC cells was established to verify the function of exosomal miR-3180-3p in vivo.

Results: We found that miR-3180-3p decreased in both NSCLC cell lines and patient tissues. Overexpression of miR-3180-3p or treatment with exosomal miR-3180-3p significantly repressed the cell proliferation and metastasis in NSCLC cell lines. Subsequently, we found miR-3180-3p performed function by downregulating FOXP4 protein expression. Furthermore, the volume and weight of nude mice tumor which expressed exosomal miR-3180-3p was significantly reduced.

Conclusion: Exosomal miR-3180-3p suppresses NSCLC progression by downregulating FOXP4 expression.

\section{Introduction}

Lung cancer is one of the most common tumors with highest mortality rate. Non-small cell lung cancer (NSCLC) accounts for $85 \%$ of all lung cancer ${ }^{[1]}$. Despite progress in treatments for NSCLC, the 5 -year survival rate is less than $15 \%^{[2-3]}$. To better understand the mechanism of NSCLC and find the potential prognostic biomarkers is still a priority.

MicroRNAs (miRNAs) are a kind of single-stranded non-coding RNAs with 19-24 nt in length, which can regulate gene expression at post-transcriptional level by targeting 3'-untraslated region (3'-UTR) of $m R N A^{[4]}$. The aberrant expression of miRNAs is closely related to cancers and tumor microenvironment ${ }^{[5]}$. MiR-421 was reported to contribute to NSCLC cell migration, invasion and inhibit cell apoptosis by mediating $\beta$-catenin-miR-421-KEAP1 axis ${ }^{[6]}$. MiR-330-3p promotes brain metastasis and epithelialmesenchymal transition (EMT) by mediating GRIA3-TGF- $\beta 1$ interaction ${ }^{[7]}$.

Exosomes are extracellular vesicles with lipid bilayer-enclosed structure which are smaller than $150 \mathrm{~nm}$. They contain proteins and nucleic acids that can be released from many cell types ${ }^{[8-9]}$. Exosomes often contain RNA molecules or proteins which provide a mechanism for these bioactive compounds to transfer into neighboring cells, thus mediating intercellular communication ${ }^{[10-11]}$. 
The RNAs concentrated in exosomes include miRNAs, mRNAs, IncRNAs (long noncoding RNAs) and circRNAs (circular RNAs) ${ }^{[12-14]}$. Increasing evidence has shown that miRNA enriched in exosomes can be shuttled to neighboring cells and regulate their biological functions ${ }^{[15,16]}$. Exosomes come from tumor cells which have been found relative to tumor angiogenesis, invasion and chemoresistance ${ }^{[17-19]}$. For example, Human mesenchymal stem cells (MSCs) derived exosomes enriched miR-143 transfer to prostate cancer cells and inhibit PC3 cell proliferation and metastasis by suppressing TFF3 protein expression ${ }^{[20]}$. Exosomes with low miR-34c-3p expression promote migration and invasion of NSCLC by upregulating integrin $\alpha 2{ }^{2} 1^{[21]}$. With increasing attention of the function of miRNA enriched in exosomes, in this study we focus on the mechanism of miR-3180-3p in exosomes.

\section{Materials}

\section{Cell culture}

All NSCLC cell lines were obtained from the Chinese Academy of Sciences (Shanghai, China). Cell lines were cultured in RPMI-1640 (Sigma) with 10\% FBS (Thermo Fisher Scientific), 100 units/mL penicillin and $100 \mu \mathrm{g} / \mathrm{mL}$ streptomycin at $37^{\circ} \mathrm{C}$ in $5 \% \mathrm{CO}_{2}$. To remove exosomes, $\mathrm{FBS}$ for cell culture was ultracentrifuged at $100,000 \mathrm{~g}$ for 10 hours.

\section{Isolation and characterization of exosomes}

After culturing cells for $48 \mathrm{~h}$ in exosome-free FBS, medium was collected, filtrated with $0.22 \mu \mathrm{m}$ filter. Exosomes were got from ultracentrifugation-based isolation techniques ${ }^{[22]}$. Briefly, the medium was centrifuged at $300 \times \mathrm{g}$ for $10 \mathrm{~min}, 2,000 \times \mathrm{g}$ for $15 \mathrm{~min}, 5,000 \times \mathrm{g}$ at $15 \mathrm{~min}$ and $12,000 \times \mathrm{g}$ for $30 \mathrm{~min}$, then the supernatant was ultra-centrifuged at $100,000 \times \mathrm{g}$ for $70 \mathrm{~min}$. Discarded the supernatant and used PBS to wash the bottom exosomes. Pelleted the exosomes by ultracentrifugation at $100,000 \times \mathrm{g}$ for $70 \mathrm{~min}$. All ultracentrifugation steps were performed at $4{ }^{\circ} \mathrm{C}$ in Beckman ultracentrifuge (TL-100). The exosomes were assessed via nanoparticle tracking analysis (NTA; ZetaView, Particle Metrix, DE), western blotting and transmission electron microscopy (TEM; JEM-1400, JEOL, Japan).

For the exosome-tracking experiments, exosomes from A549 cells were labeled by PKH67 membrane dye (Sigma-Aldrich), labeled exosomes were washed twice by medium without FBS. A549 cells were seeded into 24-well chamber, labeled exosomes were added and incubated $3 \mathrm{~h}$. Cells were fixed with $4 \%$ paraformaldehyde for $10 \mathrm{~min}$. Cells were washed thrice with PBS, and blocked with $0.1 \%$ Triton-X 100 for $1 \mathrm{~h}$, then incubated with Actin antibody at $4{ }^{\circ} \mathrm{C}$ overnight. Next day, cells were stained $20 \mu \mathrm{g} / \mathrm{mL}$ Alexa Fluor $594 \mathrm{lgG}(\mathrm{H}+\mathrm{L})$ (Life Technologies, Grand Island, NY) for $10 \mathrm{~min}$ at room temperature. Cellular nuclei were stained with DAPI. The merge images were captured using the electron microscope (LSM 710, Carl Zeiss).

\section{Dual-luciferase reporter assay}


The FOXP4 3'UTR nucleotide sequence predicted miR-3180-3p target sites and the mutation sequence were synthesized (Genewiz, Beijing, China), digested with Xhol and Notl, and cloned into the psiCHECK-2 dual luciferase vector (Promega, Madison, WI, USA) to generate the FOXP4-3'UTR wt and FOXP4-3'UTR mut. Subsequently, NSCLC cells were plated in a 24-well plate and co-transfected with $50 \mathrm{ng}$ FOXP43'UTR wt or FOXP4-3'UTR mut and 20 nM either miR-3180-3p mimics (5'-UGGGGCGGAGCUUCCGGAG-3') or miR-NC (5'-UUCUCCGAACGUGUCACGUTT-3'). After $48 \mathrm{~h}$ transfection, cells were collected, luciferase activities were measured by Dual-Luciferase Reporter assay kit (Promega) on a TD-20/20 Luminometer (Turner Designs, Sunnyvale, CA, USA).

\section{Western blotting}

Cell or exosome lysates were acquired using RIPA lysis buffer (Beyotime, Shanghai, China) and separated by $10 \%$ SDS-PAGE. Proteins were transferred onto PVDF membranes and blocked with $5 \%$ solution of nonfat milk for $1 \mathrm{~h}$. The membranes were treated with primary antibodies against FOXP4 (ab251688, Abcam) and GAPDH (\#5174, CST) overnight at $4{ }^{\circ} \mathrm{C}$. Subsequently, The membranes incubated with a secondary antibody for 1 hour at room temperature. Protein bands were detected by ECL Substrate (Tanon Science and Technology, Shanghai, China). GAPDH was used as an internal reference.

\section{qRT-PCR}

Total RNA was extracted by TRIzol (Invitrogen, CA, USA) according to the manufacture's instruction. The cDNA synthesis with reverse transcriptase (RT) was performed using a M-MLV First Strand kit (Life Technologies). MiRNA First-Strand cDNA Synthesis Kit (Thermo Fisher Scientific) was used for miRNA reverse transcriptase. The miScript SYBR Green PCR Kit (Qiagen) was used for RT-PCR reactions. Relative expression level of miRNAs or mRNA was calculated using the ${ }^{\Delta \Delta} \mathrm{Ct}$ method. GAPDH and U6 small nuclear RNA (U6 snRNA) were used for normalization of mRNA and miRNA. The primer sequences are presented in Table 1. 
Table 1

Primer Sequence for qRT-PCR

\begin{tabular}{|c|c|}
\hline Gene & Primer Sequence $\left(5^{\prime}-3^{\prime}\right)$ \\
\hline \multirow[t]{2}{*}{ miR-3180-3p } & F: GCGTGGGGCGGAGCTT \\
\hline & R: AGTGCAGGGTCCGAGGTATT \\
\hline \multirow[t]{2}{*}{ U6 } & F: AGAGCCTGTGGTGTCCG \\
\hline & R: CATCTTCAAAGCACTTCCCT \\
\hline \multirow[t]{2}{*}{ GAPDH } & F: CCTTCCGTGTCCCСАCT \\
\hline & R: GCCTGCTTCACCACCTTC \\
\hline \multirow[t]{2}{*}{ FOXP4 } & F: AGGACACGGAGGGTTCAG \\
\hline & R: TGTGGGGAGAGCTGGTG \\
\hline \multicolumn{2}{|c|}{ FOXP4, forkhead box P4; miR-3180-3p, microRNA-3180-3p; F, forward; R, reverse } \\
\hline
\end{tabular}

\section{Cell proliferation and transwell assay}

Transwell assay was performed using 24-well chambers containing membrane filter inserts. After $48 \mathrm{~h}$ of transfection, cell lines A549 and H460 were collected and resuspended in serum-free RPMI1640 medium at the cell density of $1 \times 10^{5}$ cells $/ \mathrm{mL}$. The chambers were used for migration assay and coated with Matrigel (BD Biosciences, Franklin Lakes, NJ, USA) were used for invasion assay. The upper chambers were added to $200 \mu \mathrm{L}$ cell suspension. The under layers were added $600 \mu \mathrm{L}$ RPMI1640 medium with $20 \%$ FBS. After $24 \mathrm{~h}$ chambers were fixed with $4 \%$ paraformaldehyde for 30 min, stained with $0.5 \%$ crystal violet solution for $10 \mathrm{~min}$, and washed by PBS for three times. The cells on the upper chambers were removed with a cotton swab. The transmembrane cells were counted over three random fields. The experiment was repeated 3 times.

\section{Tumor xenograft and tail vein injection}

The 24 4-week-old nude mice were divided into 2 groups with 5 mice in each group at random for tumor growth observation. $1 \times 10^{7}$ A549 cells were resuspended with $50 \%$ Matrigel. A total of $0.2 \mathrm{~mL}$ cells was subcutaneously injected into mice. To observe the effect of exosomes on tumor growth in vivo, $100 \mu \mathrm{g}$ exosomes from transfected A549 cells which transfected with miR-3180-3p mimics or negative control in $100 \mu \mathrm{L}$ PBS were were injected into mice by tail vein every 3 days according to previous reports ${ }^{[23]}$. The tumor size was measured every 7 days. After 5 weeks all nude mice were sacrificed, the tumors were removed and weighed. This study was approved and conducted by the Institutional Animal Care and Use Committee of the Soochow University. 
Significance tests and correlation analyses were performed using GraphPad Prism 5.0 (GraphPad Software). Student's $t$ test was used in the statistical analysis. $P$-value less than 0.05 was considered statistically significant.

\section{Results}

\section{Low level expression of miR-3180-3p in NSCLC patients}

We extracted non-small cell lung cancer expression profile data from the GEO database (GEO: GSE53882). We found miR-3180-3p was significantly lower than that in paired para-carcinoma tissues (Fig. 1a). Furthermore, we detected miR-3180-3p expression in NSCLC cell lines and found miR-3180-3p was significantly lower in A549 and H460 cell lines as compared to normal cell line HBE (Fig. 1b).

\section{Overexpression of miR-3180-3p suppresses proliferation, migration, and invasion in NSCLC Cells}

To explore the function of miR-3180-3p on the biological function of NSCLC cells, we transfected A549 and $\mathrm{H} 460$ cell lines with miR-NC and miR-3180-3p mimics. The proliferation, migration and invasion of NSCLC cells were examined. First, the transfection efficiency was determined by qRT-PCR. The results showed that the expression of miR-3180-3p was strikingly increased (Fig. 2c). The biological function showed that, compared with the control group, the proliferation, migration, and invasion abilities were significantly decreased in the miR-3180-3p mimic group ( $p<0.05)$ (Figs. 2d-h).

\section{FOXP4 is the target gene of miR-3180-3p}

To determine the target gene of miR-3180-3p, we used the TargetScan software to identify FOXP4 as a possible target gene (Fig. 2a). To clarify its accuracy, a dual luciferase assay was performed. A549 and H460 cells were co-transfected with miR-NC or miR-3180-3p mimics and FOXP4-3'UTR wild type (WT) or FOXP4-3'UTR mutation type (MUT) luciferase reporter vector. As shown in Fig. 2b-c, compared with the control group, miR-3180-3p mimics significantly decreased the luciferase activity in the WT group $(p<$ $0.05)$, but had no significant effect on the MUT group ( $p>0.05)$.

To further demonstrate FOXP4 is a target gene of miR-3180-3p, we detected the FOXP4 mRNA and protein expression after transfecting by miR-3180-3p mimics. The results showed that both FOXP4 mRNA and protein expression were significantly decreased (Fig. 2d-e). The FOXP4 mRNA expression was verified by TCGA database (Fig. 2f). These results suggest that FOXP4 is the target gene of miR-3180-3p.

\section{Knockdown of FOXP4 by siRNA shows the same phenotype as the effect of upregulating miR-3180-3p on NSCLC cells.}

To explore the effects of FOXP4 on NSCLC, we used FOXP4-siRNA to knock down FOXP4 in A549 and H460 cells. The protein expression level of FOXP4 was significantly reduced in the two siRNA groups compared with the control group (Fig. 3a). In order to further clarify the effects of FOXP4-siRNA on cancer cell proliferation and metastasis, we used CCK-8 and transwell assay to determine the effects of FOXP4 on NSCLC cells. CCK-8 assay revealed that the growth rate of A549 and H460 cells transfected with 
siRNA were significantly lower than control group cells (Fig. 3b). Transwell assay revealed that FOXP4 knockdown remarkably inhibited the migration and invasion of NSCLC cells (Fig. 3c-d).

\section{Overexpression of FOXP4 reverses the effects of miR-3180-3p on NSCLC cells}

Since our results suggested that miR-3180-3p inhibited cell growth and metastasis through the downregulation of FOXP4, we explored whether FOXP4 is a direct functional mediator of the inhibitory effects of miR-3180-3p. As expected, we observed that exogenous miR-3180-3p could significantly suppress the expression of FOXP4 and the FOXP4 expression was retarded after ovexpression of FOXP4 (Fig. 4a-b). Functionally, CCK-8 assays showed that miR-3180-3p could suppress the proliferation of NSCLC cells and the suppression could be blocked by overexpressed FOXP4 (Figrue 4c-d). Furthermore, Transwell migration and invasion assays showed that miR-3180-3p could inhibit the metastasis of NSCLC cells and the inhibition could be blocked by increased FOXP4 (Fig. 4e-f). Collectively, these observations suggested that miR-3180-3p could inhibit the growth and metastasis of NSCLC, at least partially, through the miR-3180-3p-FOXP4 pathway.

\section{Exosomal miR-3180-3p serves as a mediator in intercellular communication}

It is well known that exosomes play an important role in cell-to-cell communication, and they can change the physiological function of recipient cells through biological active factors ${ }^{[24]}$. From the website Exocarta we know that miR-3180-3p exist in exosomes. To further verify miR-3180-3p enriched in exosomes, we extract exosomes after transfecting miR-3180-3p mimics into A549 cells. The canonical morphology of exosomes was captured by transmission electron microscopy (TEM) which confirmed the presence of extracted exosomes (Fig. 5a). The sizes of exosomes were measured by particle size distribution. The sizes of exosomes was measured by particle size distribution with approximately $30-$ $100 \mathrm{~nm}$ diameter (Fig. 5b). The expression of exosome markers such as Annexin V, Flotillin-1 and CD63 were examined by western blot which further proved the successful isolation of exosomes from the supernatants of NSCLC cells (Fig. 5c).

\section{Exosomal miR-3180-3p inhibits proliferation, migration, and invasion in NSCLC Cells}

To determine whether the purified exosomes could enter the cells and perform functions. Exosomes derived from A549 cells were labeled with a green fluorescent marker, PKH67. The result showed that PKH67 was localized in the cytoplasm of recipient cells (Fig. 5d). QPCR determined the effective functions of exosomal miR-3180-3p. The result showed that the expression of miR-3180-3p was significantly increased in recipient NSCLC cells when treating with Exo-miR-3180-3p (Fig. 5e).

Following the previous observation that miR-3180-3p is enriched in exosomes, we supposed that miR3180-3p shuttled by exosomes could inhibit the proliferation, migration and invasion in NSCLC cells. To access the role of miR-3180-3p exosomes, NSCLC cells were treated with Exo-miR-NC or Exo-miR-3180$3 p$. The results showed that FOXP4 protein levels in A549 and H460 cells exposed to miR-3180-3p exosomes were significantly decreased compared to miR-NC exosomes (Fig. 5f). In addition, The 
proliferation was inhibited by miR-3180-3p exosomes (Fig. 5g-h). And the migratory and invasive abilities obtained similar results (Fig. 5i-j).

\section{Effect of exosomal miR-3180-3p on tumor in vivo}

To further explore the role of exosomal miR-3180-3p in NSCLC in vivo, A549 cells were subcutaneously injected into nude mice. Exosomes extracted from A549 cells transfected with miR-3180-3p or miR-NC mimics were injected to mice by tail vein. In line with our analysis in vitro, tumor tissues of nude mice injected with miR-3180-3p exosomes attenuated tumor size and weight (Fig. 6a-d).

\section{Discussion}

With the rapid development of technology, many diseases can be cured in clinic. However, there is no effective treatment for cancer. Many studies have reports that miRNAs play important roles in cancers. Accumulating evidence indicates that miRNAs are involved in the progression of NSCLC. These provides miRNAs as a promising approach to treat cancer. However, the mechanism of miR-3180-3p in the regulation of NSCLC remains unclear.

In this study, we researched the roles of miR-3180-3p in NSCLC. We found downregulation of miR-3180$3 p$ both in NSCLC cell lines and human samples. Upregulation of miR-3180-3p suppressed NSCLC cell proliferation and metastasis. We further determined that tumor oncogene FOXP4 was a direct target of miR-3180-3p.

FOXP4 belongs to Human Forkhead-box (FOX) gene family. Forkhead proteins have been proved to play key roles in cell cycle regulation and oncogenesis. Yin et al. reported microRNA-491-5p suppresses osteosarcoma cell proliferation and promotes apoptosis by targeting FOXP4 ${ }^{[25]}$. Restoration of FOXP4 expression significantly promotes breast cancer cell proliferation, migration and invasion ${ }^{[26]}$. FOXP4 upregulates in NSCLC, especial in adenocarcinoma lung cancer. We found downregulated FOXP4 prominently suppressed NSCLC cell proliferation, migration and invasion.

Exosomes are secreted by cells, circulate in the blood and induce a series of cellular reactions when being captured by recipient cells. Recent studies have suggested that tumor-derived exosomes may play an important role in tumor progression ${ }^{[27-29]}$. HCC cells release exosomes containing both SMAD3 protein and mRNA, these exosomes enhance the recipient HCC cells SMAD3 signaling and increase their adhesive ability ${ }^{[30]}$. Feng Wei et al. showed miRNAs within exosomes play important roles in cancers. For example, exosomal miR-222-3p promotes the proliferation, gemcitabine resistance, migration and invasion of NSCLC cells by targeting the promoter of SOCS3 ${ }^{[31]}$. We isolated exosomes derived from two NSCLC cell lines - A549 and H460 under overexpression of miR-3180-3p. We found miR-3180-3p can be enriched in exosomes. The overexpression of exosomal miR-3180-3p and silencing of FOXP4 both suppressed NSCLC cell proliferation, migration and invasion. 
In summary, we are the first time to demonstrate exosomal miR-3180-3p inhibited NSCLC proliferation and metastasis by targeting FOXP4. These results revealed a new regulatory network involving miR-3180$3 p$ and FOXP4, which may be applied to the therapeutic treatment of NSCLC in the future.

\section{Conclusions}

We demonstrated that the miR-3180-3p carried by exosomes could suppress NSCLC cells proliferation and metastasis by downregulating FOXP4. These findings might provide a foundation for determination of innovative therapeutic strategies.

\section{Abbreviations}

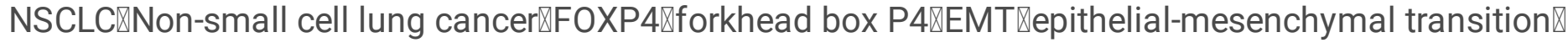
NTA囚nanoparticle tracking analysis $\triangle T \mathrm{TEM} \unrhd$ transmission electron microscopy; snRNA: small nuclear RNA; RT: reverse transcriptase; 3'-UTR: 3'-untraslated region; miRNAs: microRNAs.

\section{Declarations}

\section{Ethics approval and consent to participate}

This study was approved and conducted by the Institutional Animal Care and Use Committee of the Soochow University.

\section{Consent for publication}

Not applicable.

\section{Availability of data and materials}

The data sets used and/or analyzed during the current study are available from the corresponding author on reasonable request.

\section{Competing interests}

No potential conflicts of interest were disclosed.

\section{Funding}

This work was supported by the National Natural Science Foundation of China (81672934 and 81873417).

\section{Authors' contributions}


Designed research and supervised: JZ; Performed research: TFC and YLL; Analysis and/or interpretation of data: CL, CD and JC; Wrote the manuscript: TFC; Revising the manuscript critically for important intellectual content: HFZ. All authors read and approved the final manuscript.

\section{Acknowledgements}

Not applicable.

\section{References}

1. Siegel RL, Miller KD, Jemal A. Cancer statistics, 2018. CA Cancer J Clin. 2018;68:7-30.

2. Wood SL, Pernemalm M, Crosbie PA, Whetton AD. Molecular histology of lung cancer: from targets to treatments. Cancer Treat Rev. 2015;41:361-375.

3. Wu X, Ruan L, Yang Y, Mei Q. Analysis of gene expression changes associated with human carcinoma-associated fibroblasts in non-small cell lung carcinoma. Biol Res. 2017;50:6.

4. Bayraktar R, Van Roosbroeck K, Calin GA. Cell-to-cell communication: microRNAs as hormones. Mol Oncol. 2017;11:1673-1686.

5. Rupaimoole R, Calin GA, Lopez-Berestein G, Sood AK. miRNA Deregulation in Cancer Cells and the Tumor Microenvironment. Cancer Discov. 2016;6:235-246.

6. Duan FG, Wang MF, Cao YB, Dan L, Li RZ, Fan XX, et al. MicroRNA-421 confers paclitaxel resistance by binding to the KEAP1 3'UTR and predicts poor survival in non-small cell lung cancer. Cell Death Dis. 2019;10:821.

7. Wei C, Zhang R, Cai Q, Gao X, Tong F, Dong J, et al. MicroRNA-330-3p promotes brain metastasis and epithelial-mesenchymal transition via GRIA3 in non-small cell lung cancer. Aging (Albany NY). 2019;11:6734-6761.

8. Kahlert C, Kalluri R. Exosomes in tumor microenvironment influence cancer progression and metastasis. J Mol Med (Berl). 2013;91:431-437.

9. Kalluri R. The biology and function of exosomes in cancer. J Clin Invest. 2016;126:1208-1215.

10. Raposo G, Stoorvogel W. Extracellular vesicles: exosomes, microvesicles, and friends. J Cell Biol. 2013;200:373-383.

11. De Silva N, Samblas M, Martinez JA, Milagro FI. Effects of exosomes from LPS-activated macrophages on adipocyte gene expression, differentiation, and insulin-dependent glucose uptake. J Physiol Biochem. 2018;74:559-568.

12. Bellingham SA, Coleman BM, Hill AF. Small RNA deep sequencing reveals a distinct miRNA signature released in exosomes from prion-infected neuronal cells. Nucleic Acids Res. 2012;40:10937-10949.

13. Huang X, Yuan T, Tschannen M, Sun Z, Jacob H, Du M, et al. Characterization of human plasmaderived exosomal RNAs by deep sequencing. BMC Genomics. 2013;14:319.

14. Li Y, Zheng Q, Bao C, Li S, Guo W, Zhao J, et al. Circular RNA is enriched and stable in exosomes: a promising biomarker for cancer diagnosis. Cell Res. 2015;25:981-984. 
15. Valadi H, Ekstrom K, Bossios A, Sjostrand M, Lee JJ, Lotvall JO. Exosome-mediated transfer of mRNAs and microRNAs is a novel mechanism of genetic exchange between cells. Nat Cell Biol. 2007;9:654-659.

16. Cha DJ, Franklin JL, Dou Y, Liu Q, Higginbotham JN, Demory Beckler M, et al. KRAS-dependent sorting of miRNA to exosomes. Elife. 2015;4:e07197.

17. Wang M, Yu F, Li P, Wang K. Emerging Function and Clinical Significance of Exosomal circRNAs in Cancer. Mol Ther Nucleic Acids. 2020;21:367-383.

18. Umezu T, Tadokoro H, Azuma K, Yoshizawa S, Ohyashiki K, Ohyashiki JH. Exosomal miR-135b shed from hypoxic multiple myeloma cells enhances angiogenesis by targeting factor-inhibiting HIF-1. Blood. 2014;124:3748-3757.

19. Ramteke A, Ting H, Agarwal C, Mateen S, Somasagara R, Hussain A, et al. Exosomes secreted under hypoxia enhance invasiveness and stemness of prostate cancer cells by targeting adherens junction molecules. Mol Carcinog. 2015;54:554-565.

20. Che Y, Shi X, Shi Y, Jiang X, Ai Q, Shi Y, et al. Exosomes Derived from miR-143-Overexpressing MSCs Inhibit Cell Migration and Invasion in Human Prostate Cancer by Downregulating TFF3. Mol Ther Nucleic Acids. 2019;18:232-244.

21. Huang W, Yan Y, Liu Y, Lin M, Ma J, Zhang W, et al. Exosomes with low miR-34c-3p expression promote invasion and migration of non-small cell lung cancer by upregulating integrin alpha2beta1. Signal Transduct Target Ther. 2020;5:39.

22. Li P, Kaslan M, Lee SH, Yao J, Gao Z. Progress in Exosome Isolation Techniques. Theranostics. 2017;7:789-804.

23. Chen G, Huang AC, Zhang W, Zhang G, Wu M, Xu W,et al. Exosomal PD-L1 contributes to immunosuppression and is associated with anti-PD-1 response. Nature. 2018;560:382-386.

24. Huang X, Yuan T, Tschannen M, Sun Z, Jacob H, Du M, et al. Characterization of human plasmaderived exosomal RNAs by deep sequencing. BMC Genomics. 2013;14:319.

25. Yin Z, Ding H, He E, Chen J, Li M. Up-regulation of microRNA-491-5p suppresses cell proliferation and promotes apoptosis by targeting FOXP4 in human osteosarcoma. Cell Prolif. 2017; 50(1):e12308.

26. Circular RNA circMYO9B facilitates breast cancer cell proliferation and invasiveness via upregulating FOXP4 expression by sponging miR-4316. Arch Biochem Biophys. 2018;653:63-70.

27. Zeng Z, Li Y, Pan Y, Lan X, Song F, Sun J, et al. Cancer-derived exosomal miR-25-3p promotes premetastatic niche formation by inducing vascular permeability and angiogenesis. Nat Commun. 2018;9:5395.

28. Guo D, Lui GYL, Lai SL, Wilmott JS, Tikoo S, Jackett LA, et al. RAB27A promotes melanoma cell invasion and metastasis via regulation of pro-invasive exosomes. Int J Cancer. 2019;144:3070-3085.

29. Faict S, Muller J, De Veirman K, De Bruyne E, Maes K, Vrancken L, et al. Exosomes play a role in multiple myeloma bone disease and tumor development by targeting osteoclasts and osteoblasts. Blood Cancer J. 2018;8:105. 
30. Fu Q, Zhang Q, Lou Y, Yang J, Nie G, Chen Q, et al. Primary tumor-derived exosomes facilitate metastasis by regulating adhesion of circulating tumor cells via SMAD3 in liver cancer. Oncogene. 2018;37:6105-6118.

31. Wei F, Ma C, Zhou T, Dong X, Luo Q, Geng L, et al. Exosomes derived from gemcitabine-resistant cells transfer malignant phenotypic traits via delivery of miRNA-222-3p. Mol Cancer. 2017;16:132.

\section{Figures}
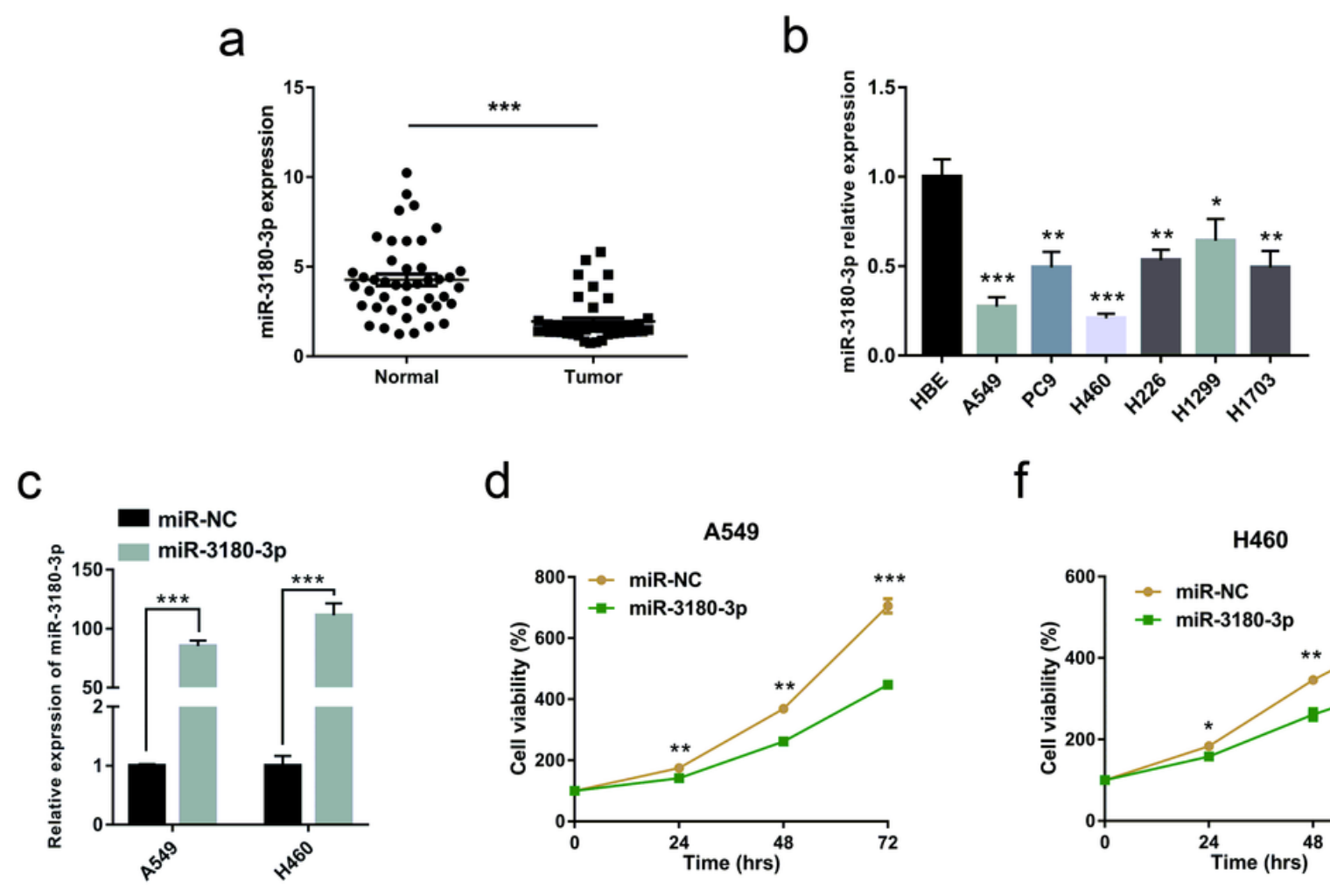

f

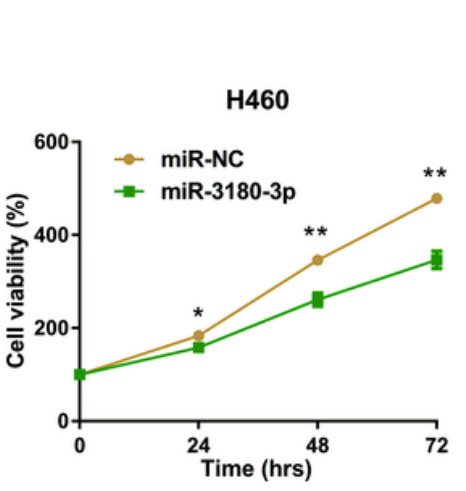

g

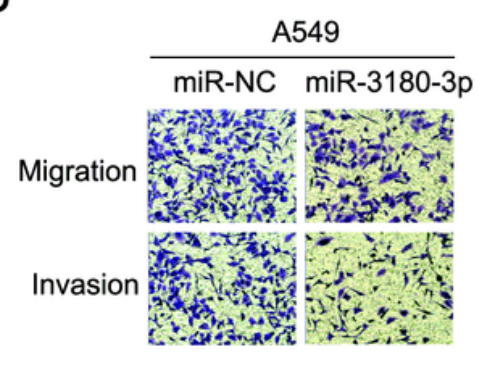

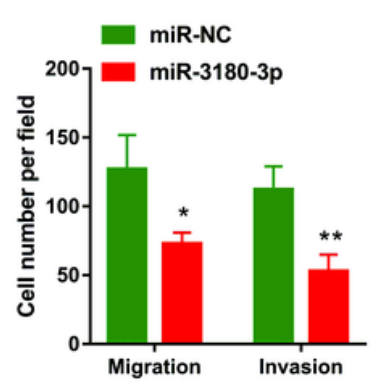

$\mathrm{h}$

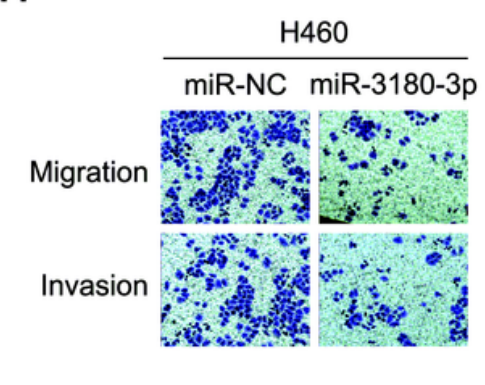

\section{Figure 1}

Overexpression of miR-3180-3p inhibits proliferation, migration and invasion in NSCLC cells. (a) The expression of miR-3180-3p in non-small cell lung cancer and para-carcinoma tissues from the GEO database (GEO: GSE53882). (b) The expression of miR-3180-3p in 6 NSCLC cell lines and a normal cell 
line. (c) The expression of miR-3180-3p after overexpression miR-3180-3p in A549 and H460 cells. (d-e) CCK-8 assay was performed in A549 and H460 cells transfected with miR-3180-3p and miR-NC. (f-g) The migratory and invasive ability of A549 and $\mathrm{H} 460$ cells transfected with miR-3180-3p and miR-NC was measured by transwell assay. ${ }^{*}<<0.05,{ }^{*} \mathrm{P}<0.01,{ }^{*} * \mathrm{P}<0.001$, and $n \geq 3$.

a

\begin{tabular}{|c|c|c|c|c|}
\hline & 636 & 711 & 674 & 1613 \\
\hline FOXP4-3'UTR wt & ACCUGGCUCCAGACCCGCCCCC. & UCCAGCCGCCCC.... & CCCCCCCGCCCCA. & GCCUCCGCCCCC \\
\hline miR-3180-3p & $\underset{\text { GAGGCCUUCGAGGCGGGGU }}{\|\|}$ & $\underset{\text { UuCGAGGCGGGGU }}{\|\|}$ & $\underset{\text { UUCGAGGCGGGGU }}{\|\|}$ & $\underset{\text { UucGAGGCGGGGU }}{\|\|}$ \\
\hline
\end{tabular}

FOXP4-3'UTR mut ACCUGGCUCCAGACGCCCGCGC...CUCCUCCAGGCCCGCG...UCUCCCCCCGCCCGCGA....AGGGGCCUGCCCGCGC

b

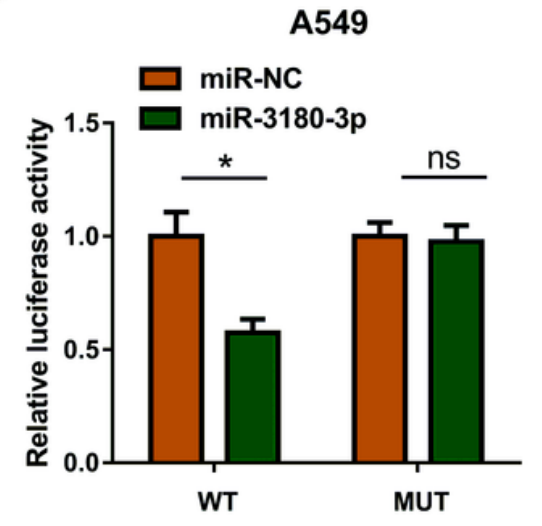

e

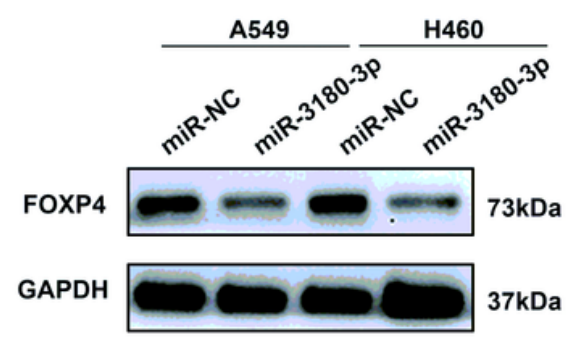

C
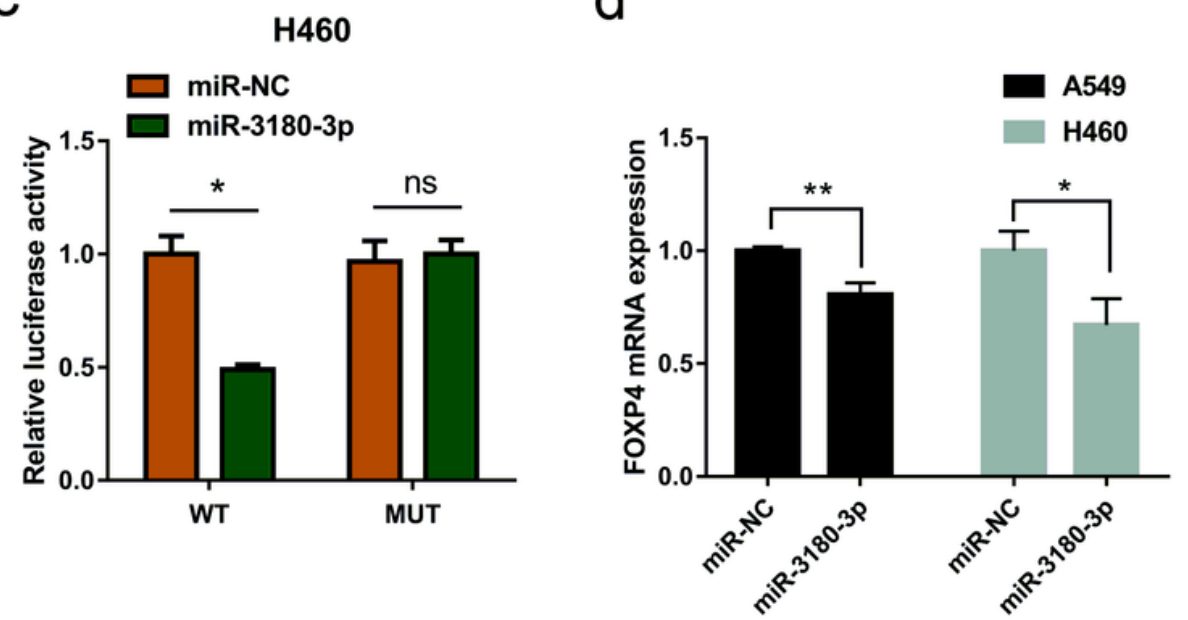

f

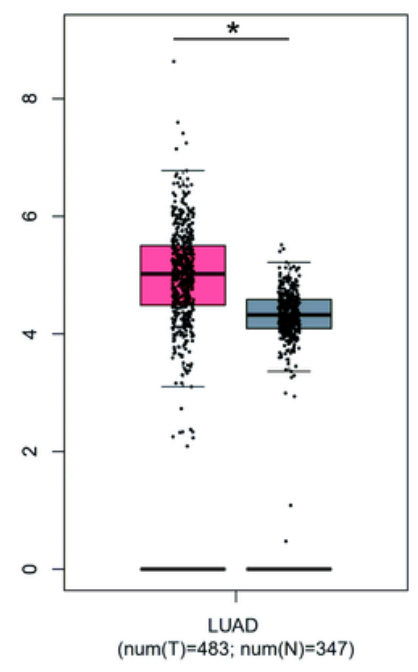

Figure 2

Identification of FOXP4 as a miR-3180-3p target gene. (a) The wild-type and a mutated type of binding site between miR-3180-3p and FOXP4. (b-c) A549 and H460 were co-transfected with miR-NC and miR3180-3p with the WT and MUT 3'UTR. Luciferase activity was measured after $24 \mathrm{~h}$ co-transfection. (d-e) The mRNA and protein level of FOXP4 was analysed by qRT-PCR and western blotting in A549 and H460 
cells. (f) The FOXP4 mRNA expression was extracted from The Cancer Genome Atlas (TCGA). *P $<0.05$, $\star \star P<0.01$, and $n \geq 3$.

a

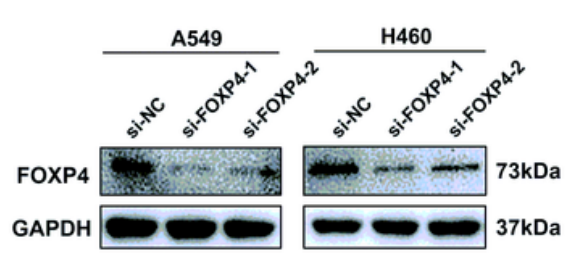

C

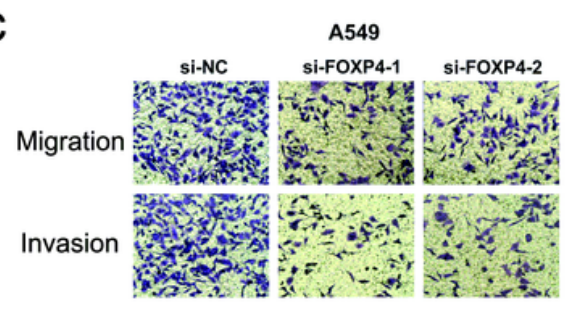

b
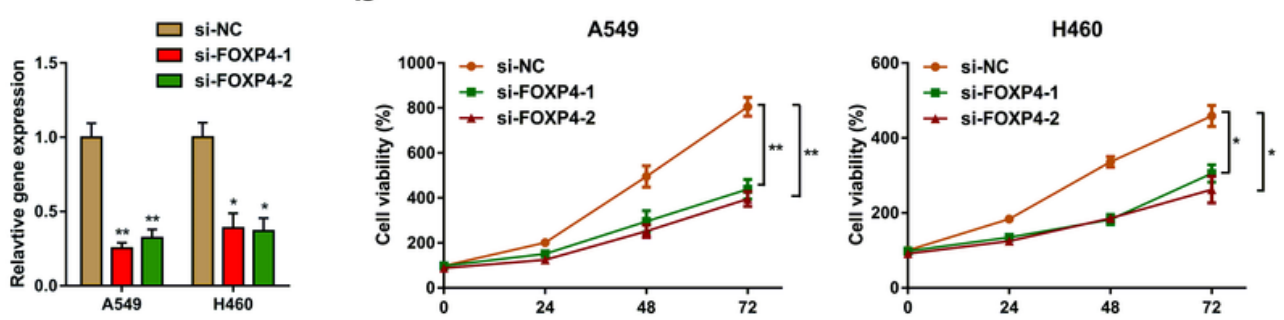

d

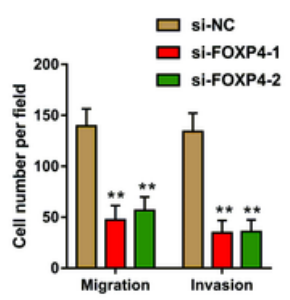

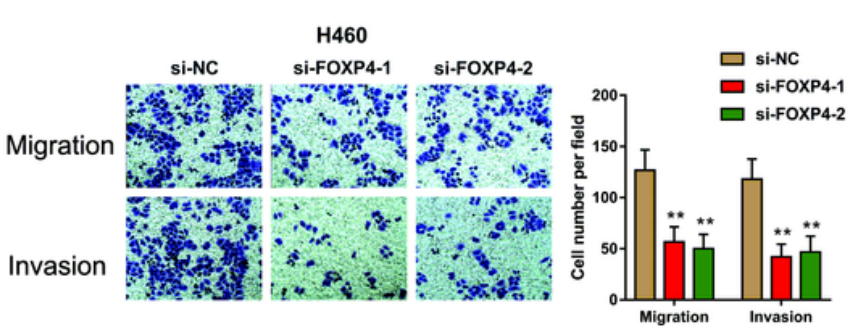

Figure 3

Knockdown FOXP4 inhibits proliferation, migration and invasion in NSCLC cells. (a) The protein level of FOXP4 in A549 and H460 cells were analysed by western blotting after transfection with siRNA target to FOXP4. (b) CCK-8 assay was performed in A549 and H460 cells after knockdown FOXP4 by siRNA. (d-e) The migratory and invasive ability of $\mathrm{A} 549$ and $\mathrm{H} 460$ cells was measured by transwell assay after knockdown FOXP4 by siRNA. *P $<0.05,{ }^{* * P}<0.01$, and $n \geq 3$. 
a

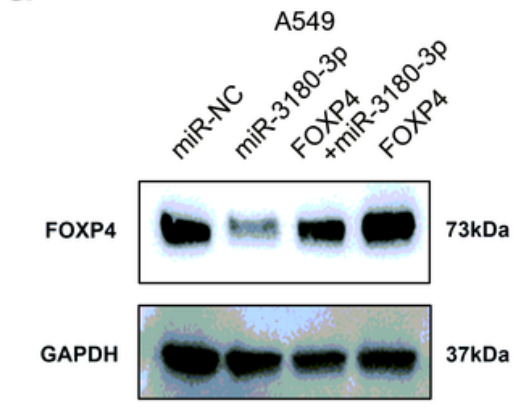

C

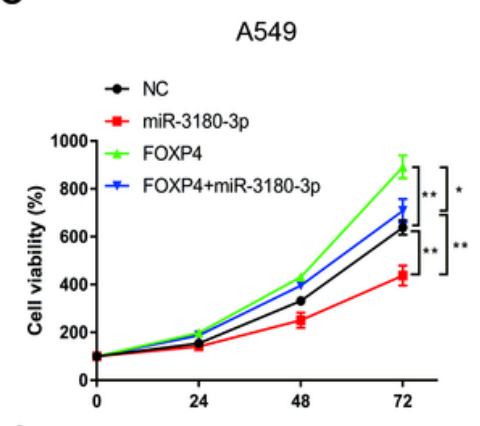

d

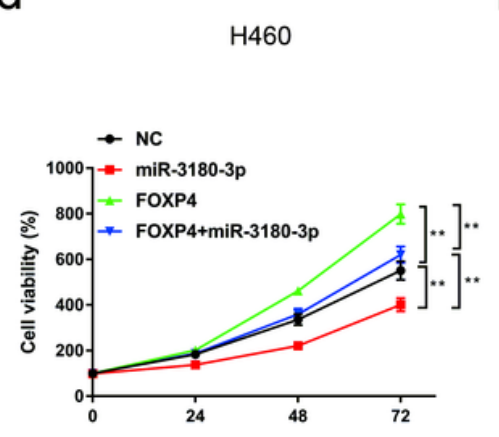

e b

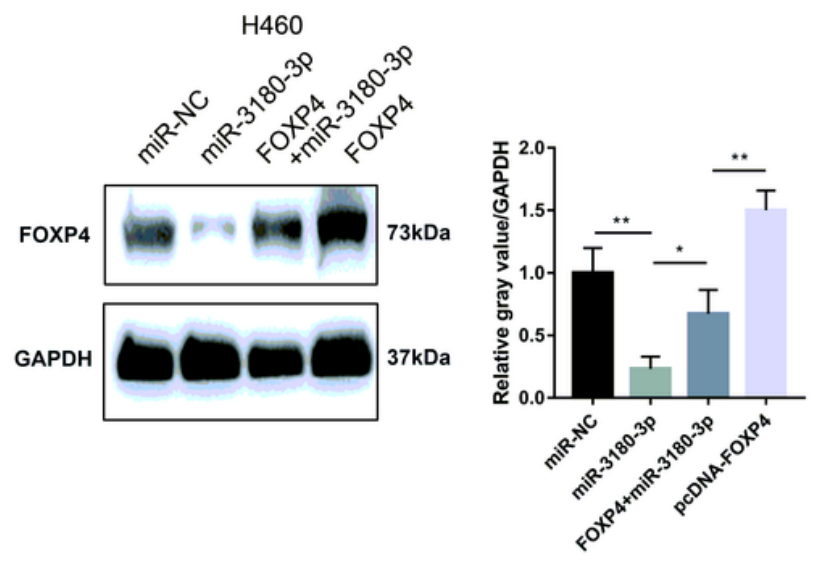

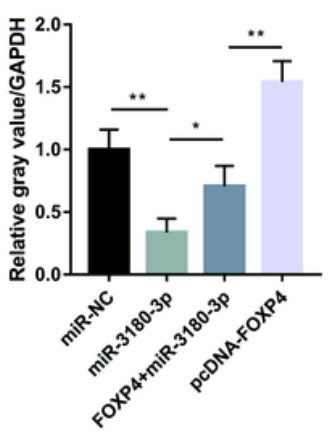

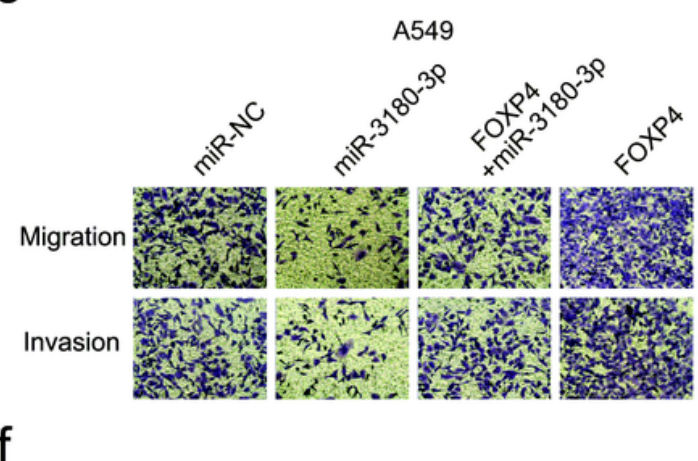

$\mathrm{H} 460$
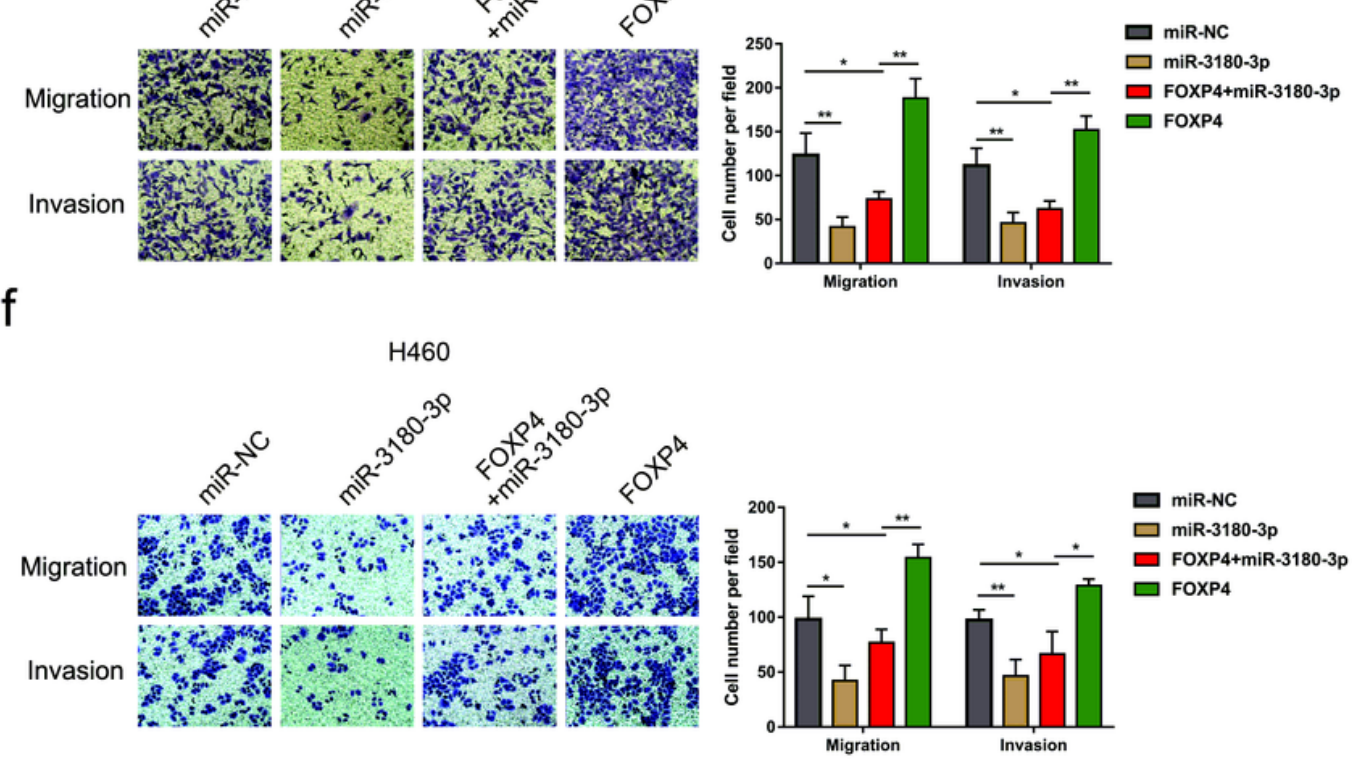

\section{Figure 4}

Partial reversal of the inhibitory effects of miR-3180-3p on the NSCLC cell phenotype by FOXP4. A549 and $\mathrm{H} 460$ cells were transfected with miR-NC, miR-3180-3p, miR-3180-3p+FOXP4 or FOXP4 respectively. $(a-b)$ western bolts were performed to dectect FOXP4 protein expression. (c-d) CCK-8 assay was performed. (e-f) The migratory and invasive ability of A549 and $\mathrm{H} 460$ cells was measured by transwell assay. ${ }^{*} P<0.05, * * P<0.01$, and $n \geq 3$. 
a

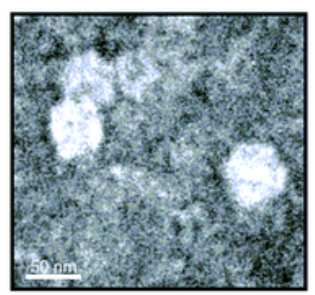

d

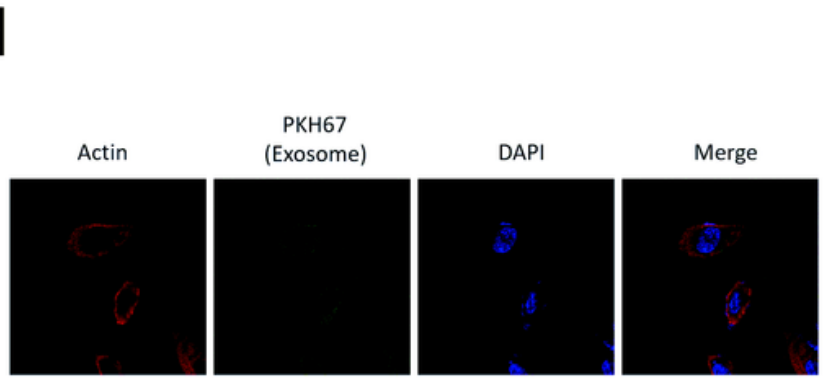

f
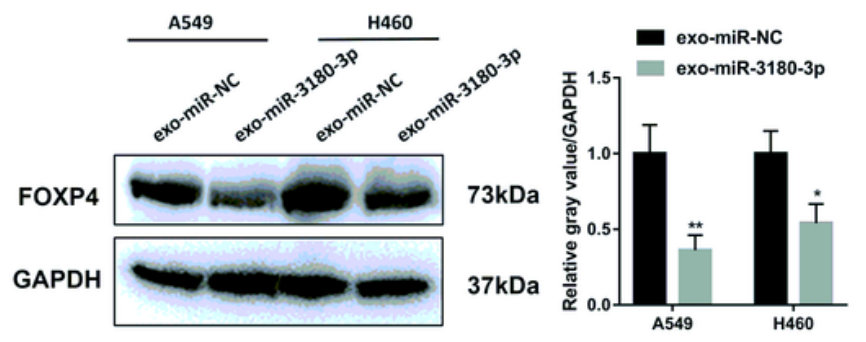

i

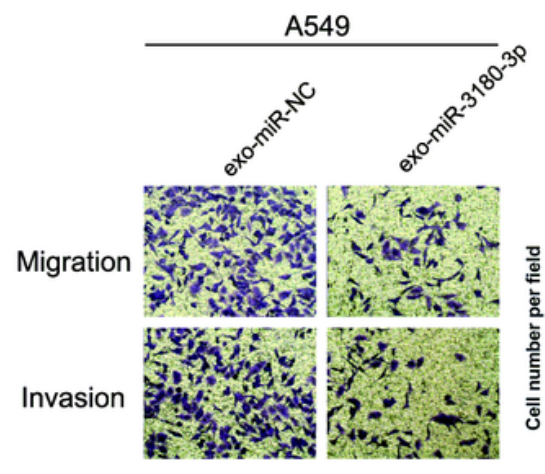

g b

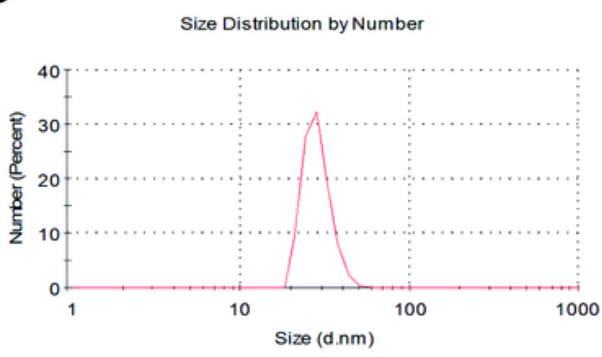

C

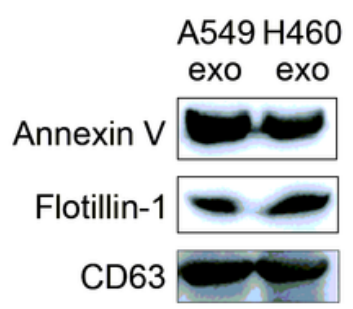

e

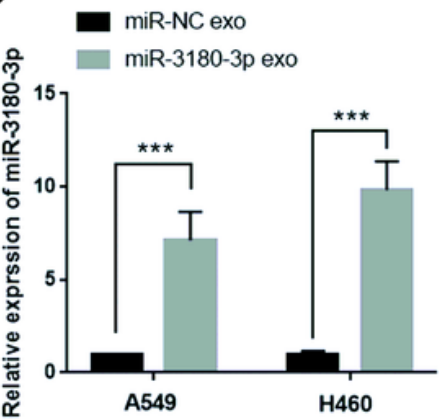

$\mathrm{h}$

A549

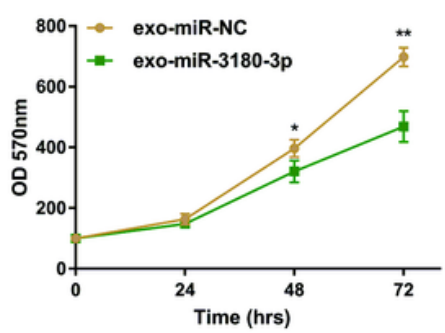

H460

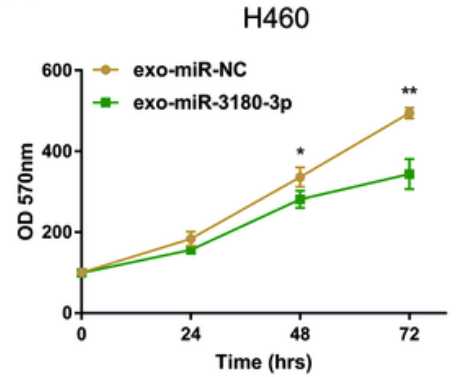

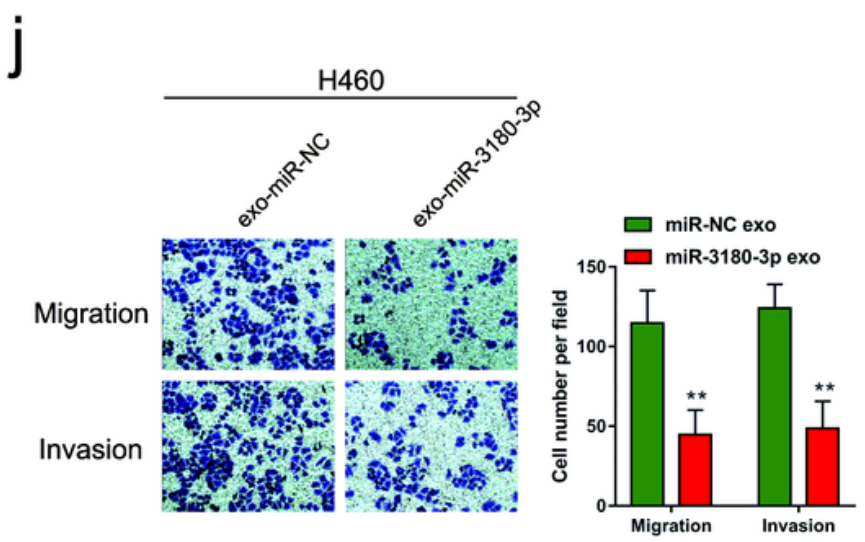

\section{Figure 5}

Exosomal miR-3180-3p derived from A549 and H460 cells suppresses proliferation and metastasis of NSCLC cells. (a) Observation of the morphology of exosomes under transmission electron microscope (scale bar, $50 \mathrm{~nm}$ ). (b) The size distribution of exosomes. (c) The expression of exosomal marker Annexin V, Flotillin-1 and CD63. (d) Representative immunofluorescence image shows the internalization of PKH67-labeled A549 - derived exosomes (green) by macrophages. Blue represents nuclear DNA staining by DAPI. Red represents cytoplasm incubation with $\beta$-actin antibody. (e) MiR-3180-3p levels in exo-A549 
and exo-H460 were measured by RT-PCR. (f) western bolts were performed. (g-h) CCK-8 assay was performed. (i-j) The migratory and invasive ability of $\mathrm{A} 549$ and $\mathrm{H} 460$ cells was measured by transwell assay. ${ }^{*} \mathrm{P}<0.05,{ }^{*} \mathrm{P}<0.01, * * * \mathrm{P}<0.001$ and $\mathrm{n} \geq 3$.

a

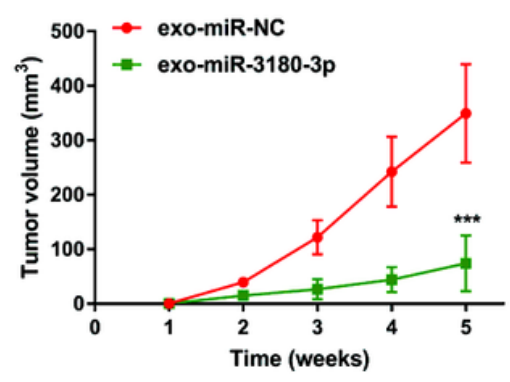

d b

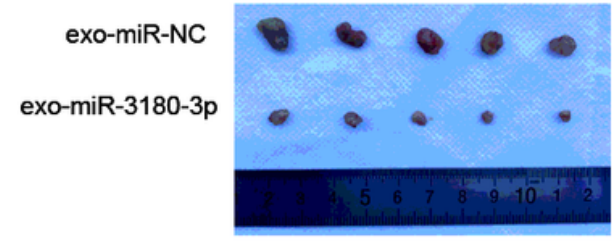

NSCLC cells

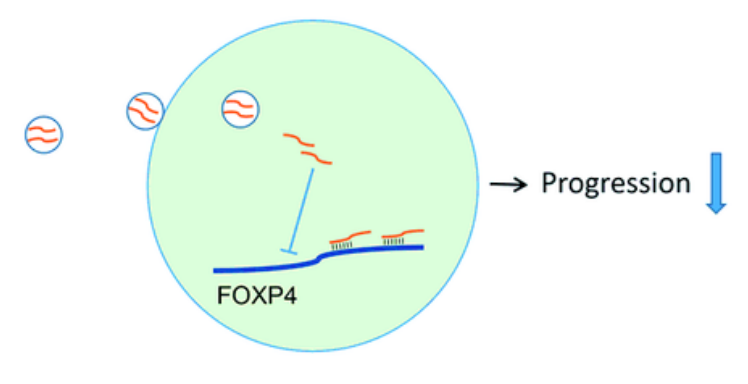

\section{Figure 6}

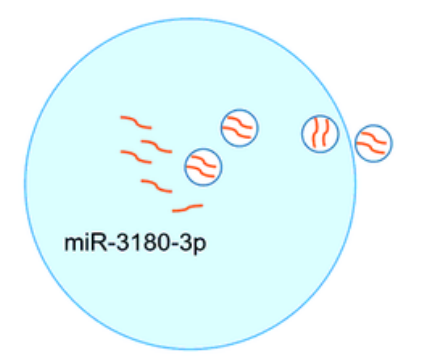

Exosomes enriched with miR-3180-3p supresses tumor growth in vivo. (a) Statistical analysis tumor volume growth curves of mice. (b-c) Photograph of tumor and curve of tumor weight. (d) Schematic model of exosomal miR-3180-3p inhibits NSCLC progression. ${ }^{\star} \mathrm{P}<0.01$, ${ }^{\star \star \star} \mathrm{P}<0.001$ and $\mathrm{n}>3$. 